\title{
USING GPS-TRACKING TECHNOLOGY FOR URBAN DESIGN INTERVENTIONS
}

\author{
S.C. van der Spek \& C.M. van Langelaar
}

Delft University of Technology, Faculty of Architecture, Department of Urbanism, Chair of Urban Design Julianalaan 134, 2628 BL Delft, The Netherlands - S.C.vanderSpek@tudelft.nl; C.M.vanLangelaar@tudelft.nl

\section{Commission IV/WG8}

KEY WORDS: tracking technologies, urban design and planning, GPS, spatio-temporal data

\section{ABSTRACT:}

The aim of this paper is to express the application of tracking technologies in Urban Design and Planning. Tracking technologies can be used to monitor different user groups in different occasions. Since 2005, TU Delft has explored and developed two types of research using GPS to collect spatio-temporal data in urban environments : (1) visitors of city centers and (2) households in specific district, specific street or specific types of buildings. The collected data can be used for analyzing, visualizing and understanding how the city functions and how people perceive the city.

\section{INTRODUCTION}

\subsection{GPS-tracking}

Can GPS-technology change the world? Probably it is not the technology itself, but unforeseen applications of the technology, which enables new features, offers new methods for research, enables collection of new types of information, or results in improving collection by up scaling the number of respondents and duration of collection. The application of new methods might lead to innovative or better insights, or validate current expec-

tations.

The Global Positioning System (GPS) was originally developed for orientation (knowing where you are) and navigation (knowing were to go). Today, providing Location Based Services (LBS) is an important feature of GPS. Nevertheless, GPS can also be used for collecting spatio-temporal data by retrieving the sequence of positioning data in space and time saved on a GPS device. In this case the GPS device is used as a data logger. This is called GPS-tracking (Spek et al, 2008).

\subsection{Time geography}

This paper is building on earlier studies on developing the existing concept of time geography in order to better understand the logic of visitor activities in time and space (Hägerstrand, 1970).
Whyte (1988) used time-lapse photography to chart the anatomy of the city. Previous research shows the value of analysing visitor behaviour using a Global Positioning Systems (GPS) tracking system (Harder Hovgesen et al., 2008).

Compared to existing methods, GPS tracking contains added values like providing accurate and valid information of quantitative spatio-temporal data. Therefore, it makes a clear contribution to the lack of knowledge about processes (movement patterns of people) while it improves static and dynamic visualisation. Changes in behaviour can be measured before, during and after investments in public space. Tourist behaviour in time and space - or geovisualisation - is extensively discussed by (Shoval \& Isaacson, 2010).

\section{MAKING THE CASE}

What is the use of collecting spatio-temporal data? As Urban Designers we would like to know more about actual processes in the city and understand better the behaviour of its visitors and inhabitants. Traditional methods are available. Firstly, traditional mapping techniques give insight in the programme and network the city offers. The programme consists of the buildings and functions inside them, most important are the attractors and the landmarks. The network consists of the public space and infrastructure. Together programme and network form the frame of our movement: the programme as initiator, attractor, the network enabling the necessary movement between origin (home) and destination (attraction). Based on the analysis of the morphology, exploring the form of our city, we can expect some movement patterns.
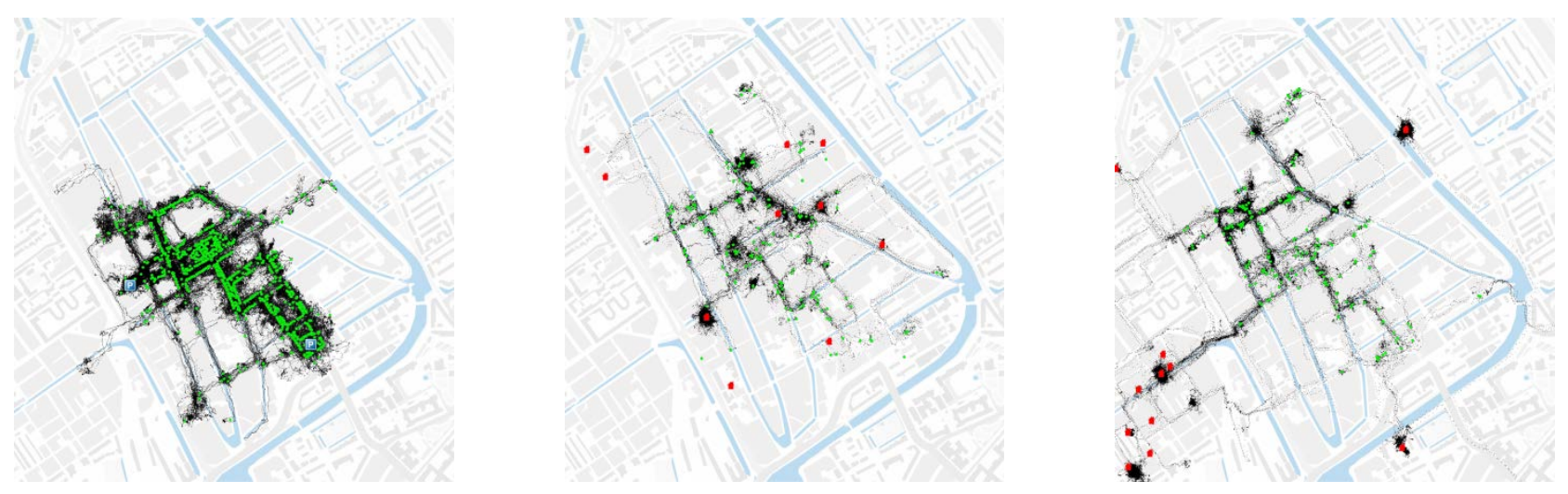

Figure 1. Tracking results of Tracking Delft: track and destinations. (left) visitors from parking garages, (middle) inhabitants of city centre, (right) inhabitants of surrounding district 

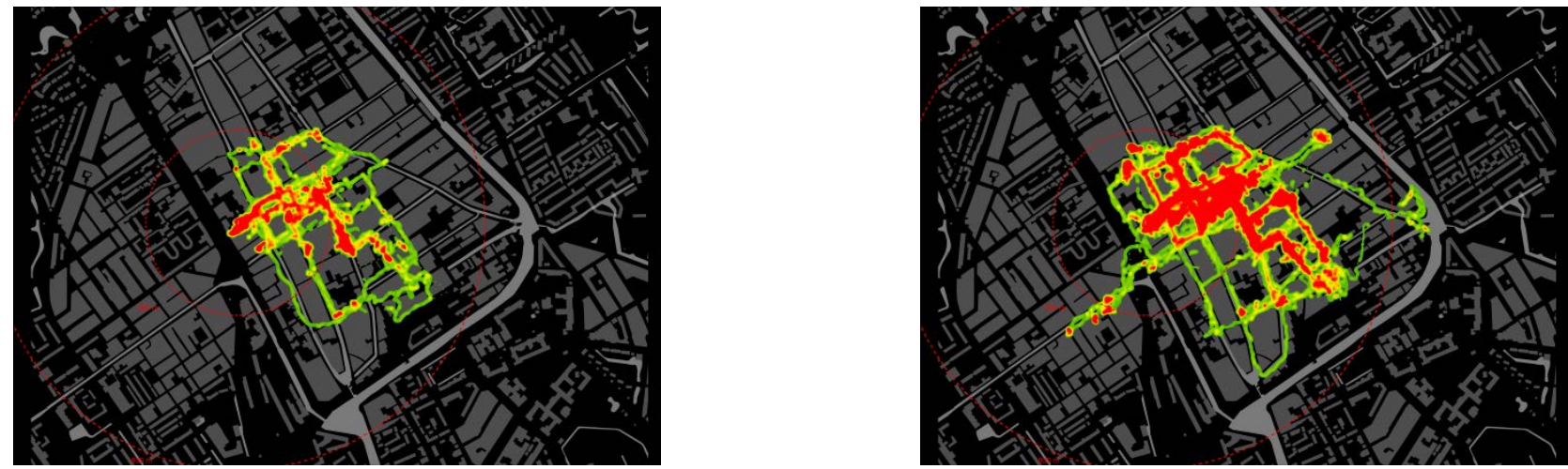

Figure 2. Tracking results of Tracking Delft (kernel density) based on origin of the visitor: unfamiliar - familiar.

Secondly, observation of visitors can be used to learn more about actual behaviour. Traditional techniques for this are monitoring specific locations, describing behaviour, counting people. These techniques are limited to a location, lack spatiotemporal information and don't provide 'the full story'.

Thirdly, interviews and diaries can be used to collect the spatiotemporal component and provide background information, e.g. on the expectations and experience. Unfortunately, we cannot fully trust the information in diaries and questionnaires provided by the participants: our brain has its limitations and people tend to give the 'best' answer.

Tracking technologies have added value to these methods by delivering accurate and reliable information on the actual activities of the respondents in space and time: based on space and time, travelled route, destination(s) and duration of stay and movement are collected. Last but not least, tracking technologies directly deliver spatial outcomes, which can easily be presented on maps or used for other types of static and dynamic visualisations.

\section{METHOD(S)}

\subsection{Data collection}

At the moment, GPS is the most accurate and reliable source for outdoor collection of spatio-temporal data on an urban scale. Other technologies are less accurate, more expensive, and less frequent or require equipment to be installed in the build environment. For GPS-tracking, only a high-sensitive receiver is needed.

Within the method developed GPS-tracking method, TU Delft distinguishes two types of research: (a) Visitors and (b) Inhabitants. These types differ in way of working, duration and target groups. Both types of researches have their own goal (Spek et al, 2009).

\subsection{Visitors (pedestrians)}

The research on Visitors concerns the collection of tracking data from an access or entrance point into a city, event or attraction. Access points can be parking garages, bicycle storages, railway stations and public transport stops, or in the case of an event or attraction the gates. Most convenient are parking garages, as people have to return to their car. From the access point, participants carry a GPS device during their walking trip. After the trip, the GPS is returned and a questionnaire is taken to collect additional, background information.

\subsection{Households (all modes)}

The research on Households concerns the collection of tracking data with as base the participant's home during a dedicated period of time. The participants can be selected based on specific households situations and on spatial requirements, such as dis- trict, street or building. For several days, the participants carry the GPS device with them during all outdoor activity. Every time the participant leaves the house and returns after an activity is defined as a round-trip. One participant produces multiple trips during the research period. Every part of the trip between destinations is defined as a leg. For every leg the mode of transportation can be determined.

During the research, the participant is responsible for charging and switching on the device. Further, the respondent will have to fill in a diary. After a specific number of days, the GPS devices are picked up and additional data is collected during an interview.

\subsection{Retrieving and Processing}

In both types of research, the GPS device is handed to participants. Data retrieval will take place after returning the device. In the next step all data is cleaned and validated. A tripdatabase including all trip-related information will be build. The database will be connected to the spatio-temporal data using the TRIPID field.

\subsection{Analysis, Visualisation and conclusions}

After the spatio-temporal data is joined with the background information, queries and calculations can be performed to analyze the data. A clear research question is essential to select the appropriate attributes and context information. The context can be defined based on the theoretical framework of the research question. The data can be analyzed on an individual level (per track) or on a generic level (per set based on a query). Conclusions can be drawn by comparing different attributes, by equating different themes or by confronting parts of the dataset with exogenous data sources.

\section{APPLICATION}

This research was initiated at TU Delft in 2005 as part of the Spatial Metro project. Here GPS-tracking was introduced as a method to evaluate the effectiveness of investments in public space by observing and measuring behaviour of pedestrians visiting the city centre. In 2007 research was carried out in the historic city centres of Norwich (UK), Rouen (F) and Koblenz (D) resulting in astonishing outcomes regarding behaviour of tourists. Further, a pilot on households was initiated in three specific districts of Almere (NL), showing the inefficiency and incompetence of the frame (public space and infrastructure).

In 2009 over 300 pedestrians were tracked in the city centre of Delft (see Fig. 1 \& Fig. 2) and twenty households were tracked in Rotterdam Charlois in the framework of Assisted Living. This resulted in the definition of specific returning types of user patterns.

In 2010 eighty people living in designated districts in Delft participated (see Fig. 1). The research resulted in a good overview of different user groups and their contribution to the city. 
Finally, in 2011 GPS-tracking was used to collect spatiotemporal data of inhabitants of high-rise buildings in Rotterdam (see Fig. 3). The results show the contribution of this group to the activities offered in the city centre.

\section{RESULTS}

In this paragraph we would like to generically emphasize on the results of applying tracking technologies in city centres and on households by TU Delft for several years now.

The first conclusion is that the different sets of data result into a rich source for data mining. Not only can the spatio-temporal information be analyzed and evaluated (A1) within the framework of the city itself, but also (A2) externally by comparing the results of different cities for specific aspects. In this case the context is different: different street pattern, different location of attractions, different landmarks.

A second crossover can be made by (B) comparing the results of different user groups within one city. Here the frame is equal (same city centre), only a variation of users has been followed. Finally, in the High-rise project three distinctions can be made based on the building and on the participants: (C1) inhabitants
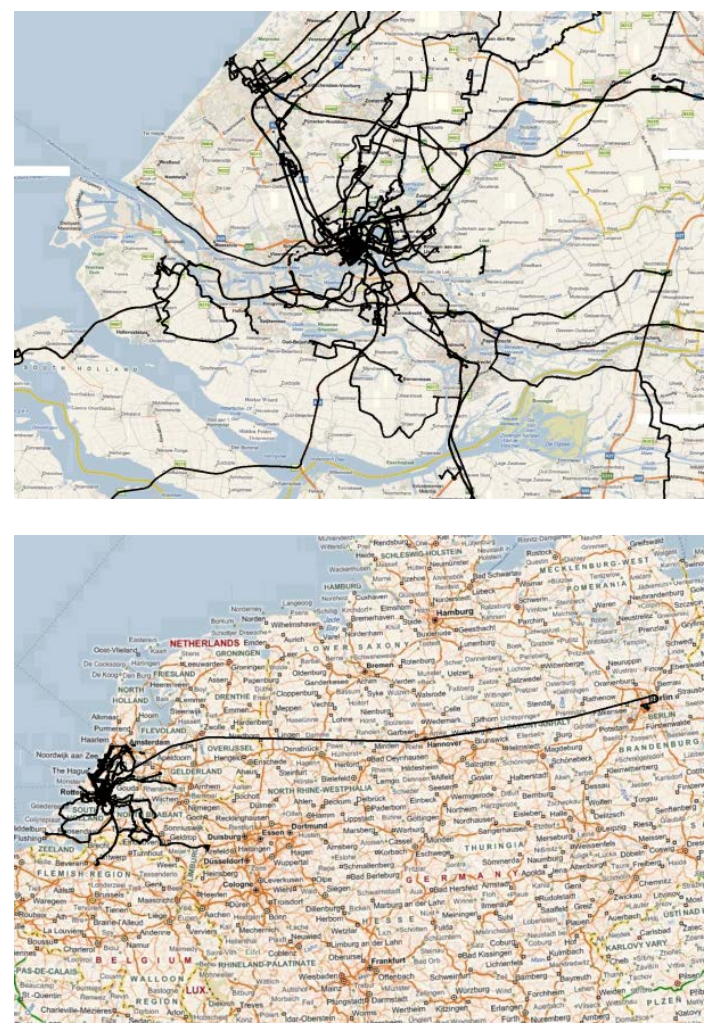

vertheless, there are also some limitations: collection, processing and the significance of results.

\subsection{Collection}

The way of distributing and returning the GPS devices is time consuming and risky: will all devices be returned? How is a representative user group selected? A good point is that the collection of data is during a fixed timespan: all data is collected at the same dates during the same conditions. The experiment is fixed in place and time. Another advantage is the easy collection of (essential) background information.

\subsection{Processing}

At the moment retrieving and validating the tracks is a time consuming part of the processing. This part can be optimized by developing tools for automatically classifying, cleaning and splitting of tracks and for recognizing stops and destinations. Manual work has to be reduced to a minimum.

\subsection{Results}

The sample of participants has to be taken into account in rela-

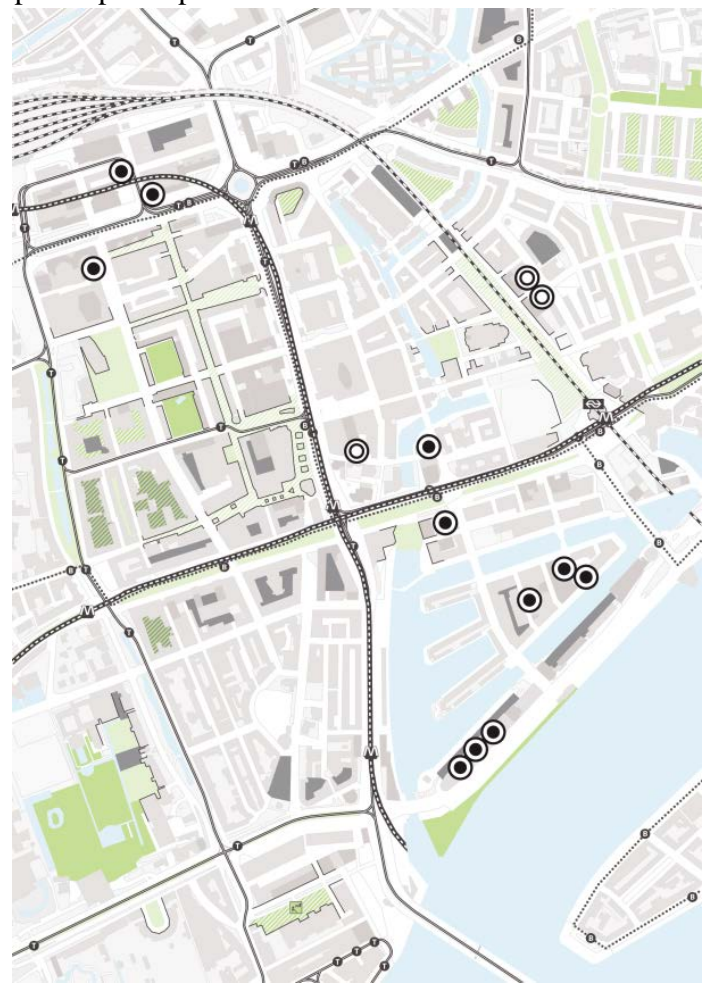

Figure 3. First tracking results of Tracking High-rise, Rotterdam, May 2011: (left, top) regional pattern, (left, bottom) (inter-) national pattern, (right) clusters of participating Highrise buildings (black dots).

of high-rise versus ground allocated housing; (C2) comparison between towers based on spatial aspects (the frame) and (C3) comparison between different lifestyle groups, e.g. depending on age, income, rental or owned apartment, duration of inhabitance.

\section{CONCLUSIONS \& FUTURE SCOPE}

In our opinion GPS-tracking technology can contribute to traditional mapping, analysis and collection techniques. GPStracking is a complementary method, providing more accurate and reliable information about activity patterns of humans. Ne- tion to the whole population. The main question is how much data is required to produce reliable results. By carrying out research from one or two access points, or from a minimal number of households in a district, street or building, the value of the outcomes is relatively low in respect to the context. To produce more significant results, especially in the case of sub sampling, larger datasets are required. Therefor, processing of the data has to be optimized, and developing new ways of collecting are essential. 


\subsection{Future: iPhone, Smartphone, regular phone?}

In the (near) future distributing and collecting devices for GPStracking might be history. Already some smartphones have the ability to function as a GPS-logger and provide the data wireless. Unfortunately, an issue is the battery capacity of Smartphones, especially while using GPS: the battery drowns in 2-3 hours. Real-time collection is not necessary for the design process. However, it could improve the efficiency of processing by automatically filling the database. Further, wireless collection enables extending the duration of the research. New ways to reach participants will be needed.

A second improvement would be using the features of a smartphone, by having it operating as interactive tool. This way, response on specific behaviour, e.g. visiting a specific location, could be generated. With data mining and location-based services in the background, the smartphone could even intervene, not only requesting feedback but also by providing feedback.

\section{ACKNOWLEDGEMENT}

The methods have been developed for Spatial Metro, an European project financed by Interreg IIIb (2005-2008). The research has been carried out in cooperation with the cities of Norwich (UK), Rouen (F), Koblenz (D), Delft (NL), Rotterdam (NL), The Hague/Scheveningen (NL). Next to the municipalities, the following partners contributed to the project: Veldacademie (Rotterdam), DOK (Delft) and Kustatelier (Delft).

\section{REFERENCES}

Bois, P.G. de. 2009. Zoetermeer anchor point research 2009: Frame - Pattern - Circuit. Delft University of Technology, Department of Urbanism

Dijst, M.J. 1995. The elliptical life: Action space as an integral measure for reach and mobility - model development with double-earning households with children living in Houten and Utrecht as example. Utrecht: Elinkwijk BV

Hägerstrand, T. 1970. What about people in regional science? Papers of the Regional Science Association, 24(1): 7-21.

Harder Hovgesen, H., Bro, P. \& Tradisauskas, N. 2008. Tracking visitors in public parks: Experiences with GPS in Denmark. In: J. van Schaick \& S.C. van der Spek (eds) Urbanism on track, Application of tracking technologies in urbanism: 6576. Amsterdam: IOS Press.

Shoval, N. \& Isaacson, M. 2010. Tourist mobility and advanced tracking technologies. New York: Routledge

Spek, S.C. van der, Schaick, J. van, Bois, P.G. de, Haan, A.R. de, 2009 Sensing Human Activity: GPS Tracking', in: Sensors, Basel, Switzerland, 2009

Spek, S.C. van der, Schaick, J. van, Hoeven, F.D. van der, Smit, M.G.J. 2008 'Urbanism on Track - Application of Tracking Technologies in Urbanism', IOS Press, Amsterdam, the Netherlands, 2008

Whyte, W.H., 1988. City: Rediscovering the center. New York: Doubleday. 